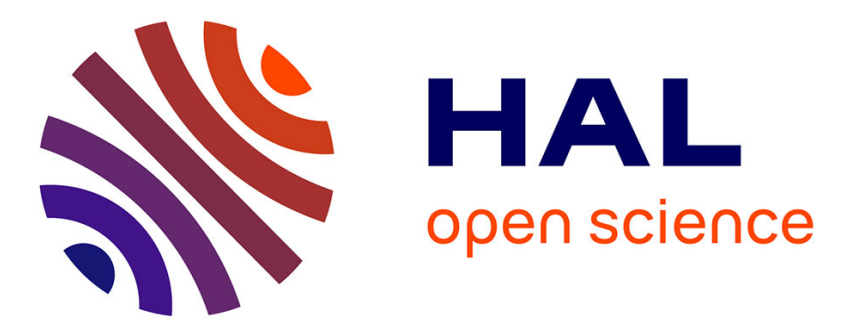

\title{
Remplacement partiel des protéines du lait par celles du blé ou du maïs dans les aliments d'allaitement : influence sur l'utilisation digestive chez le veau de boucherie
} R. Toullec, J.F. Grongnet, H. Flageul, Non Renseigné, P.M. Lucas

\section{- To cite this version:}

R. Toullec, J.F. Grongnet, H. Flageul, Non Renseigné, P.M. Lucas. Remplacement partiel des protéines du lait par celles du blé ou du maïs dans les aliments d'allaitement: influence sur l'utilisation digestive chez le veau de boucherie. Productions Animales, 1990, 3 (3), pp.201-206. hal-00895903

\section{HAL Id: hal-00895903 https://hal.science/hal-00895903}

Submitted on 1 Jan 1990

HAL is a multi-disciplinary open access archive for the deposit and dissemination of scientific research documents, whether they are published or not. The documents may come from teaching and research institutions in France or abroad, or from public or private research centers.
L'archive ouverte pluridisciplinaire HAL, est destinée au dépôt et à la diffusion de documents scientifiques de niveau recherche, publiés ou non, émanant des établissements d'enseignement et de recherche français ou étrangers, des laboratoires publics ou privés. 
INRA Prod. Anim., 1990, 3 (3), 201 - 206
R. TOULLEC et J.F. GRONGNET avec la collaboration de H. Flageul, J.Lareynie et P.M. Lucas

INRA Laboratoire du Jeune Ruminant 65, rue de Saint-Brieuc 35042 Rennes Cedex
Remplacement partiel des protéines du lait par celles du blé ou du maïs dans les aliments d'allaitement :

\author{
influence sur l'utilisation digestive \\ chez le veau de boucherie
}

L'application de la politique des quotas laitiers a provoqué une réduction drastique de la quantité de poudre de lait écrémé disponible pour la fabrication des aliments d'allaitement et de fortes fluctuations de son prix. Depuis 1988, l'octroi de la prime de dénaturation à la poudre de lait écrémé utilisée en alimentation animale n'est plus subordonné à un taux minimum d'incorporation. Il en résulte un grand intérêt pour l'emploi des protéines de substitution. Cependant, le veau préruminant est très sensible à la nature des protéines alimentaires. Le gluten de blé et les protéines de maïs sont-ils utilisables?

Certaines protéines de substitution sont peu digestibles et peuvent entraîner des refus ou des troubles digestifs. Ceux-ci se manifestent notamment par des diarrhées, des perturbations de la motricité gastrointestinale et des altérations de la paroi intestinale, induisant une augmentation de l'absorption des macromolécules (revue de Troccon et Toullec, 1989). Les troubles se développent en même temps que les titres d'anticorps dirigés contre les protéines alimentaires. Ils disparaissent lorsque les protéines incriminées sont retirées du régime et

\section{Résumé}

Le but de cet essai était d'étudier l'influence du remplacement d'une partie des protéines du lait par celles du blé ou du maïs, sur la digestibilité de l'aliment et la rétention azotée, chez le veau de boucherie. Pour cela, 4 laits de remplacement ( $T, B V, B D$ et M) ont été distribués à des lots de 6 veaux entre les âges d'environ 4 et 19 semaines. Dans l'aliment $\mathrm{T}$, les protéines étaient apportées en totalité par de la poudre de lait écrémé. Dans les autres aliments, $19 \%$ des protéines étaient fournies soit par du gluten de blé vital (BV) ou dévitalisé (BD), soit par un concentrat protéique de maïs (M). La digestibilité apparente et la rétention azotée, mesurées au cours des $8^{\mathrm{e}}$ et $14^{\mathrm{e}}$ semaines d'âge, n'ont pas été significativement affectées par la nature des protéines de l'aliment : en moyenne, la digestibilité de l'azote a été de $0,95,0,95,0,95$ et 0,93 pour les aliments $T, B V$, BD et $M$, respectivement. Les valeurs calculées ont été de $0,94,0,93$ et 0,86 pour la digestibilité de l'azote des produits BV, BD et $M$. Les veaux des lots BV, BD et M ont développé des anticorps systémiques contre les protéines étudiées. Cependant, aucune de ces dernières ne semble avoir entraîné de réactions allergiques. La dévitalisation n'a pas eu d'influence notable sur l'utilisation du gluten de blé par les animaux, mais elle a permis d'améliorer son maintien en suspension. réapparaissent dès l'ingestion d'un repas d'épreuve distribué après quelques jours de régime d'exclusion. Ces différentes caractéristiques indiquent qu'il peut s'agir de réactions d'hypersensibilité de nature allergique. Les troubles ont été principalement observés dans le cas de certains produits issus du soja, mais ont également été décrits avec un aliment dont les protéines provenaient en totalité du gluten de blé (Kilshaw et Slade 1982). Cependant, à notre connaissance, l'utilisation d'aliments contenant des taux modérés de gluten n'a pas fait l'objet de publications.

Le but de cet essai était d'étudier l'influence du remplacement d'une partie des protéines du lait par celles du blé ou du maïs sur la digestibilité de constituants de l'aliment et la rétention azotée, chez le veau de boucherie. Les effets antigéniques éventuels des protéines testées ont été caractérisés en déterminant l'évolution des titres d'anticorps dirigés contre elles. Leur influence sur la perméabilité intestinale aux macromolécules a été appréciée d'après les variations du taux circulant de $\beta$ lactoglobuline immunoréactive.

\section{1 / Conditions expérimentales}

\section{1 / Aliments}

Quatre aliments d'allaitement (T, BV, BD et $\mathrm{M})$, contenant $21 \%$ de matières azotées et $19 \%$ de lipides, ont été préparés. Dans l'aliment T, les matières azotées étaient apportées presque 
exclusivement par de la poudre de lait écrémé. Dans les 3 autres aliments, $19 \%$ des matières azotées étaient fournies soit par du gluten de blé vital (BV) ou dévitalisé (BD), soit par un concentrat protéique de maïs $(\mathrm{M})$, le reste provenant de la poudre de lait écrémé et des acides aminés de synthèse (tableau 1).

Le gluten de blé natif est insoluble dans l'eau, mais s'hydrate facilement en formant une pâte viscoélastique. Il est préparé industriellement à partir de la farine, en utilisant sa viscoélasticité en milieu aqueux pour faciliter sa séparation de l'amidon. Le produit obtenu est appelé vital s'il est capable de reprendre ses caractères originaux au contact de l'eau et dévitalisé dans le cas contraire. La dévitalisation peut être réalisée, par exemple, à l'aide d'un traitement thermique. Le concentrat protéique de maïs est un co-produit de l'extraction de l'amidon. L'amidon et les protéines insolubles sont mis en suspension dans l'eau, puis séparés par centrifugation et séchés. La fraction protéique obtenue est dépourvue des propriétés de viscoélasticité.
Les produits étudiés sont riches en protéines mais pauvres en minéraux (tableau 2), ce qui facilite leur introduction dans les aliments d'allaitement; seule leur teneur en fer est susceptible de poser des problèmes dans le cas du veau de boucherie. Par rapport aux protéines du lait, celles des glutens de blé contiennent autant d'arginine et sont plus riches en acides aminés soufrés (tableau 3); en revanche, elles sont moins pourvues en thréonine, valine, isoleucine, leucine et surtout lysine. Les protéines de maïs sont plus riches que celles du lait en acides aminés soufrés et surtout en leucine, mais sont moins pourvues en tous les autres acides aminés indispensables et semi-indispensables, phénylalanine + tyrosine exceptées. Cependant, compte tenu des supplémentations en lysine et en méthionine (ainsi qu'en thréonine dans le cas des aliments contenant les glutens de blé), les 4 aliments utilisés ont eu des teneurs en acides aminés indispensables et semi-indispensables voisines.

Un léger dépôt se produisait au bout de 2 à $3 \mathrm{mn}$ dans le lait de remplacement préparé à

Tableau 1. Formules des aliments d'allaitement expérimentaux (\%).

\begin{tabular}{|lcccc|}
\hline \multicolumn{1}{c}{ Aliment } & T & BV & BD & M \\
\hline Poudre de lait écrémé & 59,7 & 48,3 & 48,3 & 48,2 \\
Gluten de blé vital (BV) (1) & - & 5,49 & - & - \\
Gluten de blé dévitalisé (BD) (1) & - & - & 5,45 & - \\
Concentrat protéique de maïs (M) (1) & - & - & - & 6,83 \\
Matières grasses (2) & 19,0 & 19,3 & 19,3 & 19,0 \\
DL méthionine & 0,129 & 0,129 & 0,129 & $\mathbf{0 , 0 9 9}$ \\
L lysine HCl & - & 0,362 & 0,362 & $\mathbf{0 , 3 7 6}$ \\
Thréonine & - & 0,041 & 0,041 & - \\
Complément (3) & 2,54 & 3,80 & 3,80 & 3,78 \\
Maltodextrines (4) & 3,6 & 3,7 & 3,7 & 2,9 \\
Dextrose & 4,5 & 0,9 & 0,9 & 1,9 \\
Lactose & 10,5 & $\mathbf{1 8 , 0}$ & 18,0 & 16,9 \\
\hline
\end{tabular}

(1) Société Roquette, 62136 Lestrem (BV: Viten ${ }^{\mathrm{R}}$, BD : Deviten ${ }^{\mathrm{R}}$ ).

(2) Suif $(85 \%)$ et matières grasses butyriques $(15 \%)$.

(3) Minéraux, vitamines, sorbitol et antibiotiques.

(4) Peu hydrolysées.

Tableau 2. Composition chimique des sources de protéines étudiées et des aliments d'allaitement (rapportée à la poudre).

\begin{tabular}{|c|c|c|c|c|c|c|c|c|}
\hline \multirow{2}{*}{\multicolumn{2}{|c|}{ Produit }} & \multicolumn{3}{|c|}{ Source de protéines } & \multicolumn{4}{|c|}{ Aliment } \\
\hline & & \multirow{2}{*}{$\frac{\text { BV }}{6,8}$} & \multirow{2}{*}{$\frac{\text { BD }}{5,8}$} & \multirow{2}{*}{$\begin{array}{c}\mathbf{M} \\
8,2\end{array}$} & \multirow{2}{*}{$\frac{\mathrm{T}}{3,4}$} & \multirow{2}{*}{$\begin{array}{c}\text { BV } \\
3,3\end{array}$} & \multirow{2}{*}{$\begin{array}{c}\text { BD } \\
3,2\end{array}$} & \multirow{2}{*}{$\frac{\mathbf{M}}{3,5}$} \\
\hline Humidité & {$[\%]$} & & & & & & & \\
\hline $\mathrm{N} \times 6,25$ & $"$ & 74,7 & 76,6 & 61,1 & 21,4 & 21,1 & 21,2 & 21,1 \\
\hline Lipides & $"$ & 5,1 & 5,9 & 4,2 & 18,9 & 20,1 & 20,1 & 19,8 \\
\hline Cendres & $"$ & 0,97 & 0,76 & 1,7 & 6,0 & 6,3 & 6,3 & 6,3 \\
\hline $\mathrm{Ca}$ & $"$ & 0,12 & 0,11 & 0,01 & 1,10 & 1,04 & 1,04 & 1,07 \\
\hline $\mathrm{P}$ & $"$ & 0,21 & 0,20 & 0,57 & 0,75 & 0,76 & 0,76 & 0,78 \\
\hline $\mathrm{K}$ & $"$ & 0,17 & 0,08 & 0,23 & 1,07 & 1,08 & 1,08 & 1,08 \\
\hline $\mathrm{Na}$ & $"$ & 0,01 & $<0,01$ & 0,02 & 0,43 & 0,46 & 0,46 & 0,41 \\
\hline $\mathrm{Cl}$ & $"$ & 0,14 & 0,09 & 0,10 & 0,84 & 0,87 & 0,87 & 1,07 \\
\hline $\mathrm{Zn}$ & ppm & 35 & 46 & 22 & 91 & 105 & 106 & 101 \\
\hline $\mathrm{Cu}$ & $"$ & 7 & 7 & 13 & 13 & 6 & 6 & 9 \\
\hline $\mathrm{Fe}$ & $"$ & 80 & 85 & 95 & 10 & 23 & 23 & 19 \\
\hline Mn & $"$ & 17 & 26 & 6 & 29 & 32 & 33 & 27 \\
\hline
\end{tabular}


Tableau 3. Composition en acides aminés des protéines étudiées et des aliments d'allaitement $(\mathrm{g} / 16 \mathrm{~g}$

\begin{tabular}{|c|c|c|c|c|c|c|c|}
\hline \multirow{2}{*}{ Produit } & \multicolumn{3}{|c|}{ Source de protéines } & \multicolumn{4}{|c|}{ Aliment } \\
\hline & BV & BD & $\mathbf{M}$ & $\mathbf{T}$ & BV & BD & $\mathbf{M}$ \\
\hline A. aspartique & 4,1 & 3,3 & 5,5 & 7,6 & 6,5 & 6,3 & 6,7 \\
\hline Thréonine & 2,8 & 2,6 & 3,4 & 4,3 & 4,1 & 4,0 & 4,3 \\
\hline Sérine & 4,6 & 4,4 & 5,0 & 5,4 & 5,5 & 5,4 & 5,5 \\
\hline A. glutamique & 33,8 & 37,3 & 20,5 & 21,1 & 22,5 & 23,3 & 19,7 \\
\hline Proline & 10,8 & 12,2 & 7,6 & 9,0 & 8,9 & 9,2 & 8,7 \\
\hline Glycine & 3,4 & 3,4 & 2,6 & 2,0 & 2,1 & 2,1 & 2,0 \\
\hline Alanine & 2,8 & 2,6 & 8,0 & 3,2 & 3,1 & 3,0 & 4,1 \\
\hline Valine & 4,2 & 4,0 & 4,4 & 6,0 & 5,5 & 5,4 & 5,8 \\
\hline Cystine & 2,7 & 2,8 & 2,1 & 1,1 & 1,4 & 1,4 & 1,3 \\
\hline Méthionine & 1,8 & 1,9 & 2,5 & 2,8 & 2,8 & 2,9 & 2,7 \\
\hline Isoleucine & 4,0 & 3,8 & 3,8 & 4,7 & 4,7 & 4,7 & 4,7 \\
\hline Leucine & 7,1 & 6,9 & 14,7 & 9,4 & 8,7 & 8,7 & 10,1 \\
\hline Tyrosine & 3,2 & 2,8 & 3,9 & 4,3 & 4,3 & 4,2 & 4,4 \\
\hline Phénylalanine & 4,9 & 5,4 & 5,7 & 4,6 & 4,3 & 4,4 & 4,5 \\
\hline Lysine & 2,4 & 1,8 & 1,6 & 7,9 & 7,8 & 7,6 & 8,0 \\
\hline Histidine & 2,0 & 2,0 & 1,8 & 2,6 & 2,4 & 2,4 & 2,4 \\
\hline Arginine & 3,6 & 3,5 & 2,9 & 3,4 & 3,3 & 3,3 & 3,1 \\
\hline
\end{tabular}

partir de l'aliment BV. En revanche, les laits obtenus à partir des 3 autres aliments restaient homogènes. La dévitalisation a donc permis d'améliorer le maintien en suspension du gluten de blé.

\section{2 / Animaux et mesures}

Vingt-quatre veaux mâles Holstein ont été achetés à l'âge d'environ 8 j et ont reçu un lait de remplacement classique, dont les protéines étaient exclusivement d'origine laitière, jusqu'à la fin de la 4 " semaine de présence. Ils ont alors été répartis en 4 lots de 6 et sont passés en $3 \mathrm{j}$ à l'un des 4 aliments expérimentaux T, BV, BD ou M.

La digestibilité des constituants des aliments et le bilan de l'azote, du calcium et du phosphore ont été mesurés en $7^{*}$ et $13^{\circ}$ semaine de présence, après une semaine d'adaptation en cages à bilans. Le reste du temps, les veaux ont été munis d'une muselière et logés en cases individuelles sur litières de paille. Lors de chaque semaine de mesures, les fèces et les urines ont été collectées en totalité et échantillonnées pendant $5 \mathrm{j}$ consécutifs. Les fèces ont été lyophylisées.

Des prises de sang ont été effectuées sur héparine dans une veine jugulaire externe, $5 \mathrm{~h}$ après le repas du matin, le jour qui a précédé le début de la transition, puis au milieu et à la fin de chacune des 9 semaines suivantes. Les plasmas ont été conservés à - $18^{\circ} \mathrm{C}$ jusqu'aux analyses. La $\beta$ lactoglobuline immunoréactive a été dosée dans chaque échantillon. Les titres d'anticorps dirigés contre les protéines solubles des trois produits étudiés ont été déterminés dans les plasmas prélevés avant la transition et après $1,3,5,7$ et 9 semaines de régime expérimental. Les techniques ELISA utilisées ont été décrites par Nunes do Prado et al (1989).
Les quantités d'aliment d'allaitement distribué ont augmenté progressivement de $1090 \mathrm{~g} / \mathrm{j}$ en 4" semaine de présence à 2800 à partir du début de la $16^{\circ}$ semaine. Les animaux ont été pesés une fois par semaine. L'abattage a eu lieu $123 \mathrm{j}$ après l'entrée en étable.

\section{2 / Résultats}

\section{1 / Appétit, croissance et état sanitaire}

L'appétit des animaux a été très satisfaisant dans le lot $\mathrm{T}$ puisque seul un de ses veaux a refusé plus de $1 \%$ de sa ration (tableau 4). Les refus ont été un peu plus importants dans les lots BD, $\mathrm{M}$ et surtout BV (respectivement 2, 2 et 3 veaux ont refusé plus de $1 \%$. L'un des veaux du lot $M$ n'a pu être utilisé lors de la seconde période de bilans, à cause d'une mauvaise adaptation en cage. Le gain de poids vif des animaux a été inférieur (respectivement de $3,8,2,5$ et $5,2 \%$ ) et l'indice de consommation supérieur (respectivement de 1,9,2,0 et 4,8\%) chez les veaux des lots BV, BD et $M$ par rapport à ceux du lot T. De même, le poids de carcasse a été moins élevé (de 3,8, 2,5 et $3,3 \mathrm{~kg}$ ). Cependant, aucune de ces différences n'a été significative. Les notations de coloration et d'état d'engraissement des carcasses ont été identiques pour les 4 lots, mais celles de conformation ont été moins satisfaisantes pour les lots $\mathrm{BV}, \mathrm{BD}$ et $\mathrm{M}$.

\section{2 / Digestibilité et bilans}

Comme prévu, la digestibilité apparente des aliments n'a pas évolué significativement avec l'âge des veaux, sauf pour les matières minérales, en particulier, le calcium et le phosphore, pour lesquels les valeurs ont baissé de la 
Tableau 4. Appétit, croissance, efficacité alimentaire et résultats d'abattage [moyennes et écarts-types résiduels (ETR)].

\begin{tabular}{|c|c|c|c|c|c|}
\hline Aliment & $\mathbf{T}$ & BV & BD & $\mathbf{M}$ & ETR \\
\hline Nombre de veaux & 6 & 6 & 6 & 6 & \\
\hline $\begin{array}{l}\text { Poids vif }(\mathrm{kg}) \\
\quad \text { - arrivée } \\
\text { - fin transition (1) } \\
\text { - abattage (2) }\end{array}$ & $\begin{array}{r}48,2 \\
65,5 \\
181,8\end{array}$ & $\begin{array}{r}47,7 \\
65,8 \\
177,7\end{array}$ & $\begin{array}{r}47,5 \\
65,8 \\
179,3\end{array}$ & $\begin{array}{r}47,5 \\
66,7 \\
177,0\end{array}$ & $\begin{array}{l}4,2 \\
4,5 \\
9,3\end{array}$ \\
\hline Gain de poids vif $(\mathrm{g} / \mathrm{j})(3)$ & 1251 & 1204 & 1220 & 1186 & 84 \\
\hline $\begin{array}{l}\text { Quantité d'aliment d'allaitement (kg) (3) } \\
\text { - ingérée totale } \\
\text { - refusée totale (4) } \\
\text { - ingérée / kg de gain de poids vif }\end{array}$ & $\begin{array}{r}212,3 \\
0,9 \\
1,83\end{array}$ & $\begin{array}{r}207,1 \\
6,3 \\
1,86\end{array}$ & $\begin{array}{r}210,9 \\
2,2 \\
1,86\end{array}$ & $\begin{array}{r}210,5 \\
2,8 \\
1,91\end{array}$ & $\begin{array}{l}5,6 \\
5,5 \\
0,1\end{array}$ \\
\hline Poids de carcasse chaude $(\mathrm{kg})$ & 111,1 & 107,3 & 108,6 & 107,8 & 5,9 \\
\hline $\begin{array}{l}\text { Classement (conformation) (5) } \\
-\mathrm{R} \\
-\mathrm{O}\end{array}$ & $\begin{array}{l}\mathbf{5} \\
1\end{array}$ & $\begin{array}{l}3 \\
3\end{array}$ & $\begin{array}{l}2 \\
4\end{array}$ & $\begin{array}{l}4 \\
2\end{array}$ & \\
\hline
\end{tabular}

(1) $30 \mathrm{j}$ après l'arrivée.

(2) $123 \mathrm{j}$ après l'arrivée.

(3) Entre la fin de la transition et l'abattage.

(4) Nombre de veaux ayant refusé plus de $1 \%$ de leur ration : Lot $\mathrm{T}: 1(1,3 \%)$

Lot BV : $3(12,4,2,6$ et $1,6 \%)$

Lot BD : $2(3,2$ et $2,0 \%)$

Lot $M: 2$ (4,5 et $2,1 \%)$

(5) Tous les veaux ont été classés en 1 pour la coloration et en 3 pour l'état d'engraissement.

Tableau 5. Digestibilité apparente des aliments $(\times 100)$ [moyennes et écarts-types résiduels (ETR)].

\begin{tabular}{|c|c|c|c|c|c|c|}
\hline \multirow{2}{*}{ Constituant } & \multirow{2}{*}{$\begin{array}{c}\text { Période } \\
\text { (1) }\end{array}$} & \multicolumn{4}{|c|}{ Aliment } & \multirow{2}{*}{ ETR } \\
\hline & & $\mathrm{T}$ & BV & BD & $\mathbf{M}$ & \\
\hline Matière organique & $\begin{array}{l}1 \\
2\end{array}$ & $\begin{array}{l}97,4 \\
96,7\end{array}$ & $\begin{array}{l}96,9 \\
96,5\end{array}$ & $\begin{array}{l}97,1 \\
96,3\end{array}$ & $\begin{array}{l}96,2 \\
95,5\end{array}$ & $\begin{array}{l}1,2 \\
0,9\end{array}$ \\
\hline Azote & $\begin{array}{l}1 \\
2\end{array}$ & $\begin{array}{l}95,5 \\
94,8\end{array}$ & $\begin{array}{l}94,8 \\
95,2\end{array}$ & $\begin{array}{l}95,2 \\
94,4\end{array}$ & $\begin{array}{l}93,5 \\
93,3\end{array}$ & $\begin{array}{l}1,9 \\
1,4\end{array}$ \\
\hline Matières grasses & $\begin{array}{l}1 \\
2\end{array}$ & $\begin{array}{l}95,0 \\
94,3\end{array}$ & $\begin{array}{l}93,7 \\
93,3\end{array}$ & $\begin{array}{l}94,4 \\
92,4\end{array}$ & $\begin{array}{l}92,5 \\
91,8\end{array}$ & $\begin{array}{l}2,9 \\
2,6\end{array}$ \\
\hline$\underset{* *}{\text { Cendres }}$ & $\begin{array}{l}1 \\
2\end{array}$ & $\begin{array}{l}93,9 \\
89,4\end{array}$ & $\begin{array}{l}92,4 \\
91,4\end{array}$ & $\begin{array}{l}92,4 \\
90,1\end{array}$ & $\begin{array}{l}90,7 \\
88,3\end{array}$ & $\begin{array}{l}2,9 \\
3,0\end{array}$ \\
\hline$\underset{* \star}{\text { Calcium }}$ & $\begin{array}{l}1 \\
2\end{array}$ & $\begin{array}{l}93,8 \\
86,4\end{array}$ & $\begin{array}{l}90,1 \\
87,7\end{array}$ & $\begin{array}{l}90,7 \\
86,2\end{array}$ & $\begin{array}{l}87,6 \\
85,6\end{array}$ & $\begin{array}{l}4,1 \\
3,6\end{array}$ \\
\hline$\underset{\star}{\text { Phosphore }}$ & $\begin{array}{l}1 \\
2\end{array}$ & $\begin{array}{l}98,5 \\
93,9\end{array}$ & $\begin{array}{l}97,4 \\
96,7\end{array}$ & $\begin{array}{l}97,1 \\
95,7\end{array}$ & $\begin{array}{l}94,7 \\
95,1\end{array}$ & $\begin{array}{l}1,7 \\
2,9\end{array}$ \\
\hline
\end{tabular}

(1) Périodes 1 et $2: 7^{\circ}$ et $13^{\circ}$ semaine de présence, respectivement.

${ }^{* *},{ }^{*}$ Effet période significatif $\left({ }^{* *} \mathrm{P} \leqq 0,01 ;{ }^{*} \mathrm{P} \leqq 0,05\right)$

période 1 à la période 2 (tableau 5). Cela est dû au fait que les besoins en calcium diminuent et que la régulation de sa rétention a lieu au niveau digestif. Aucune différence significative n'a pu être mise en évidence entre les 4 régimes, bien que les valeurs aient eu tendance à être un peu moins élevées avec l'aliment $M$ (pour l'azote 0,93 au lieu de 0,95 avec les aliments T, BV et BD). En admettant que la digestibilité apparente de l'azote du lait et des acides aminés de synthèse soit la même dans les 4 aliments, les valeurs calculées pour l'azote des produits $B V, B D$ et $M$ seraient de $0,94,0,93$ et
0,86 respectivement. Ces valeurs sont élevées, en particulier celles concernant les 2 glutens de blé qui sont identiques à celles observées pour les meilleurs concentrats protéiques de lactosérum (Grongnet et al 1981).

Les quantités d'azote, de calcium et de phosphore retenus par jour ont été voisines dans les 4 lots (en moyenne: $40,5,38,6,40,2$ et $39,5 \mathrm{~g}$; $20,4,18,8,18,6$ et $19,0 \mathrm{~g} ; 10,8,11,6,11,2$ et $11,1 \mathrm{~g}$, respectivement dans les lots $\mathrm{T}, \mathrm{BV}, \mathrm{BD}$ et $\mathrm{M}$ ). Seules les différences concernant le calcium entre le lot T et les lots BV et BD ont été significatives $(\mathrm{P} \prec 0,05)$. 


\section{3 / Titres d'anticorps et perméabilité intestinale aux macromolécules}

Dans le plasma des veaux du lot $\mathrm{T}$, des titres d'anticorps de 3 à 4 ont été trouvés tout au long de l'essai contre les extraits des trois produits étudiés (figure 1). Ces valeurs, qui n'ont pas évolué avec l'âge, sont probablement dues essentiellement à des attachements non spécifiques des immunoglobulines en solution peu diluée sur les plaques ELISA. Chez les veaux des lots BV, BD et $M$, les valeurs initiales ont été très voisines de celles observées chez les animaux du lot $\mathrm{T}$, mais ont augmenté significativement par la suite. Les différences par rapport au lot $\mathrm{T}$ ont généralement été significatives à partir de la $5^{\mathrm{e}}$ semaine de régime. Les protéines étudiées ont donc eu une activité antigénique. Cependant, aucun symptôme d'activité allergénique n'a pu être mis en évidence. Ainsi, le taux circulant de $\beta$-lactoglobuline immunoréactive est toujours demeuré à la limite du seuil de détection (environ $20 \mathrm{ng} / \mathrm{ml}$ ). Il ne semble donc pas y avoir eu d'accroissement de la perméabilité intestinale aux macromolécules, comme cela a été observé avec du tourteau de soja cuit, de l'ovalbumine (Kilshaw 1981) et du pois cru (Nunes do Prado et al 1988). Kilshaw et Slade (1982) ont pourtant constaté des altérations de la paroi intestinale avec du gluten de blé, mais ce demier apportait la totalité des protéines de l'aliment.

\section{Conclusion}

Dans nos conditions expérimentales, les protéines des deux glutens de blé étudiés ont eu une utilisation digestive pratiquement aussi élevée que les protéines du lait. La dévitalisation a permis d'améliorer le maintien en suspension du gluten de blé, mais n'a pas eu d'effet sur la digestibilité. La valeur obtenue pour la digestibilité des protéines issues du maïs, bien que moins élevée, est demeurée satisfaisante. Malgré la formation d'anticorps circulants dirigés contre les trois préparations étudiées, aucune ne semble avoir entraîné de réaction allergique, bien que l'importance des refus ai été accrue, en particulier avec le gluten de blé vital. Des essais complémentaires effectués sur un plus grand nombre d'animaux devraient permettre de mieux préciser l'influence de ces produits sur l'appétit, l'état sanitaire et la croissance.

\section{Références bibliographiques}

GRONGNET J.F., PATUREAU-MIRAND P., TOULLEC R. PRUGNAUD J., 1981. Utilisation des protéines du lait et du lactosérum par le jeune préruminant. Influence de l'âge et de la dénaturation des protéines du lactosérum. Ann. Zootech., 30, 443-464.

KILSHAW P.J., 1981. Gastrointestinal hypersensitivity in the preruminant calf. In Bourne F.J. Current tropics in veterinary medicine and animal science. Vol. 12. The mucosal immune system. 203-223, Martinus Nijhoff, The Hague.

KILSHAW P.J., SLADE H., 1982. Villus atrophy and crypt elongation in the small intestine of preruminant calves fed with heated soyabean flour or wheat gluten. Res. Vet. Sci., 33, 305-308.
Figure 1. Evolution des titres d'anticorps dirigés contre les protéines solubles (extrait total dans du tampon phosphate à $\mathrm{pH} 7,4$ ) des produits étudiés (nombre de dilutions au demi à partir d'une dilution initiale des plasmas au vingtième) (moyennes et écarts-types des moyennes).

$O$ veaux recevant l'aliment $T$.

- veaux recevant l'aliment contenant le produit étudié (1).

$A, B, a, b$. Dans un lot donné, les valeurs accompagnées de lettres différentes sont significativement différentes, d'après le test de Friedman (majuscules $P \leqq 0,01$ minuscules $p \leqq 0,05$ ).

${ }^{\star \star}$, ${ }^{\star}$ Différence significative entre lots $\left({ }^{* \star} P \leqq 0,01 ;{ }^{\star} P\right.$ $\leqq 0,05)$.

\section{Titre}

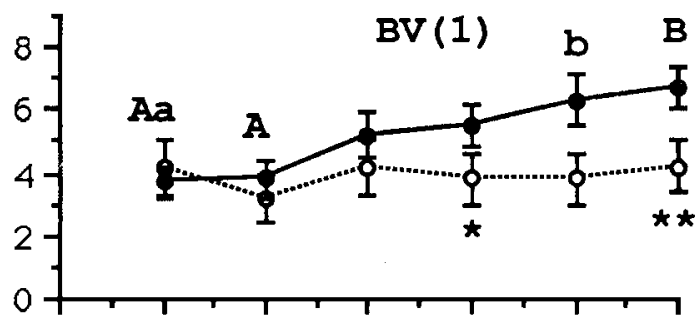

\section{Titre}

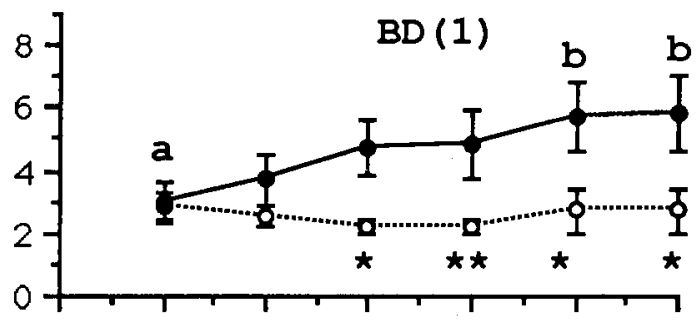

\section{Titre}

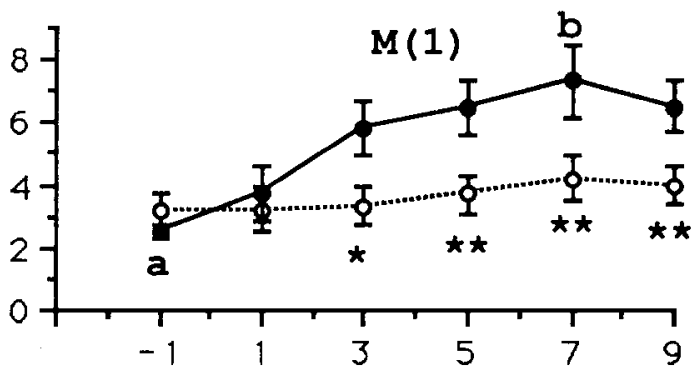

Temps depuis le début de la transition (semaines).
NUNES DO PRADO I., TOULLEC R, LALLES J.P., GUEGUEN J., HINGAND L., GUILLOTEAU P., 1989. Digestion des protéines de pois et de soja chez le veau préruminant. I. Taux circulants de nutriments, formation d'anticorps et perméabilité intestinale aux macromolécules. Reprod. Nutr. Dévelop., 29, 413-424.

NUNES DO PRADO I., TOULLEC R., LALLES I.P., HINGAND L., GUEGUEN Y., 1988. Anticorps contre les protéines alimentaires et perméabilité intestinale aux macromolécules chez le veau préruminant recevant de la farine de pois. Reprod. Nutr. Dévelop., 28, 1157-1158.

TROCCON J.L., TOULLEC R., 1989. Aliments d'allaitement pour veaux d'élevage. Remplacement de la poudre de lait écrémé par d'autres sources protéiques. INRA Prod. Anim., 2, 117-128. 


\section{Remerciements}

à la Société Roquette, 62136 Lestrem, pour l'aide apportée à la réalisation de cet essai.

\section{Summary}

Partial replacement of milk proteins by wheat or maize in milk feed: the influence on digestibility in the veal calf.

The aim of this experiment was to study the effect of the partial replacement of milk protein by wheat or maize protein on digestibility and nitrogen balance in the veal calf. Four milk-substitutes ( $\mathrm{T}, \mathrm{BV}, \mathrm{BD}$ and $\mathrm{M}$ ) were given to groups of 6 preruminant calves between 4-19 weeks of age. In the T diet, protein was entirely provided by skim milk powder. In the other diets, $19 \%$ of the protein was provided either by wheat gluten (vital : BV or devi- talized : BD) or by a maize protein concentrate (M). The apparent digestibility and nitrogen balance, measured during the $8^{\text {th }}$ and the $14^{\text {th }}$ week of age, were not significantly altered by the source of dietary protein : the mean values for nitrogen digestibility were $0.95,0.95,0.95$ and 0.93 with the $T, B V, B D$ and $M$ diets, respectively. The calculated values for the digestibility of nitrogen provided by $\mathrm{BV}, \mathrm{BD}$ and $M$ products were $0.94,0.93$ and 0.86 . Circulatory antibodies were developped by the calves of $B V, B D$ and $M$ groups against the corresponding proteins. However, none of them appeared to induce allergic responses. Devitalization had no effect on the utilization of wheat gluten by the calves, but improved its suspensibility.

TOULLEC R., GRONGNET I.F. 1990. Remplacement partiel des protéines du lail par celles du blé ou du maïs dans les aliments d'allaitement : influence sur l'utilisation digestive chez le veau de boucherie INRA Prod. Anim., 3 (3), 201-206. 